\title{
A Hopf Resonator for 2-D Artificial Cochlea: Piecewise Linear Model and Digital Implementation
}

\author{
Moslem Nouri, Arash Ahmadi, Member, IEEE, \\ Shahpour Alirezaee, Gholamreza Karimi, Majid Ahmadi, Fellow, IEEE, and Derek Abbott, Fellow, IEEE
}

\begin{abstract}
The mammalian auditory system is able to process sounds over an extraordinarily large dynamic range, which makes it possible to extract information from very small changes both in sound amplitude and frequency. Evidently, response of the cochlea is essentially nonlinear, where it operates within Hopf bifurcation boundaries to maximize tuning and amplification. This paper presents a set of piecewise linear (PWL) and multiplierless piecewise linear (MLPWL1 and MLPWL2) active cochlear models, which mimic a range of behaviors, similar to the biological cochlea. These proposed models show similar dynamical characteristics of the Hopf equation for the active nonlinear artificial cochlea. Accordingly, a compact model structure is proposed upon which a 2-D cochlea is developed. The proposed models are investigated, in terms of their digital realization and hardware cost, targeting large scale implementation. Hardware synthesis and physical implementation on a FPGA show that the proposed models can reproduce precise active cochlea behaviors with higher performance and considerably lower computational costs in comparison with the original model. Results indicate that the MLPWL1 model has a lower computational overhead, precision, and hardware cost, while the PWL model has a higher precision and dynamically tracks the original model. On the other hand, the MLPWL2 model outperforms the others in terms of accuracy, dynamical tracking of the original model and implementation cost. The gain variations of the original, PWL, MLPWL1, and MLPWL2 models are 230, 100, 105, and $230 \mathrm{~dB}$, respectively. The mean normalized root mean square errors (NRMSEs) of the PWL, MLPWL1, and MLPWL2 models are $0.11 \%, 11.97 \%$, and $0.34 \%$, respectively, as compared to the original cochlear model.
\end{abstract}

Index Terms-Active cochlea, basilar membrane (BM), Hopf bifurcation.

\section{INTRODUCTION}

$\mathbf{T}$ HE ROLE OF THE middle ear is to transmit sound vibrations from tympanic membrane to the cochlea [1]. Frequency analysis, compression of dynamic-range and amplification are three major signal processing functions of the cochlea [2]. Converting mechanical vibrations along the base to the apex of the basilar membrane (BM) into neural

Manuscript received July 30, 2014; revised October 21, 2014; accepted January 05, 2015. Date of current version March 27, 2015. This paper was recommended by Associate Editor Z. Zhang.

M. Nouri, A. Ahmadi and G. Karimi are with the Department of Electrical Engineering, Razi University, 67149-67346 Kermanshah, Iran (e-mail: moslemnouri70@gmail.com; aahmadi70@gmail.com; ghkarimi@razi.ac.ir).

S. Alirezaee and M. Ahmadi are with the Department of Electrical and Computer Engineering, University of Windsor, Windsor, ON N9B 3P4, Canada (e-mail: s.alirezaee@uwindsor.ca; ahmadi@uwindsor.ca).

D. Abbott is with School of Electrical \& Electronic Engineering, the University of Adelaide, SA 5005, Australia (e-mail: derek.abbott@adelaide.edu.au).

Color versions of one or more of the figures in this paper are available online at http://ieeexplore.ieee.org.

Digital Object Identifier 10.1109/TCSI.2015.2390555 impulses is one function of the cochlea that can be processed by the auditory nervous system [3]-[5]. In addition, it has remarkable filter functions and provides spatial separation of frequency information in a manner similar to that of a spectrum analyzer [5], [6]. In [7], Gold hypothesized electromechanical transduction in the middle ear. Transporting neural information from cochlear synapses to inner hair cells (IHCs) is carried out by the auditory nerve fibers [3], [8]. On the other hand, fibers that are connected to outer hair cells (OHCs) are clearly not affected by the sound. Thus, OHCs do not directly take part in neural coding [3]; however, recent studies have clarified the importance of OHCs for hearing as a source of the electromechanical energy [9]. Astonishingly, these sensory cells have a motor function. As a result of their motile characteristics [10], $\mathrm{OHCs}$ affect the BM and, consequently, neural information is transported by the auditory nerve fibers. The OHCs improve or amplify the vibration of the BM in a frequency-selective way at low stimulus levels. Moreover, the degree of amplification is reduced using the simultaneous presence of a second tone at a different frequency [11], [12]. There is strong evidence that the dynamic range of the cochlear is due to a Hopf bifurcation as a result of self-tuned oscillations of the OHCs [13].

Hearing aids and cochlear implants are well-known devices for individuals who suffer a cochlear hearing loss [3]. The first electronic cochlea has been provided by Lyon and Mead in 1988 [8]. Electronic cochleas have been successfully used in analog signal processing applications such as spatial localization [14], slope detection [15], computer peripheral and amplitude modulation (AM) detection [16], [17], correlation [18] and speech recognition [19]. A 2-D model, which describes an active nonlinear cochlea based on Hopf differential equations for a single variable, is presented in [20]. Analog implementation of this model using an automatic quality factor control (AQC) loop strategy is described [21]. An analog VLSI implementation of the inner hair cell [22], using circuit analogies for analysis of cochlear models [23], a time-discrete nonlinear cochlear model implemented on a DSP chip for auditory studies [24], tuning the Hopf cochlea towards listening [25] and a floating active inductor based CMOS cochlear filter have been considered [26]. Recent research on the cochlea motivates the need for models with adequate linkage between active amplification by the outer hair cells (OHCs) and the passive traveling wave along the membrane [24]. This led Kern and Stoop to deduce a biophysically detailed, energy-based model with Hopf-type amplifiers [24]. Subsequently, they presented an analog Hopf cochlear circuit with a simplified coupling pattern showing the desired accuracy [27] and [28]. The Hopf equation based model reproduces the main features of the cochlea response 
adequately in simulations [29]. In general, analog and digital implementations are used for the realization of a cochlear implant, each has benefits and drawbacks. Advantages of analog implementation are lower area and power consumption. Digital implementations, on the other hand, consume more silicon area and power per function compared to analog counterparts, but their development time is lower and are less sensitive to the power supply variation, noise or device mismatch. Digital implementation is considered to be suitable choice with high dynamic range, greater stability, and repeatability.

This study is considered a set of cochlear models, which are based on the piecewise linear approximations of the corresponding nonlinear differential equations, and their low cost digital implementation. The presented models can replace the resonator in [30]. With this approach a compact two dimensional (2-D) $N$-point artificial cochlea is implemented and without the use of the filter bank structure. Exploiting high speed digital computation capabilities and resource sharing techniques, an architecture for large scale implementation is presented and physically evaluated. The rest of this paper is organized as follows. Section II deals with the background of the topic. The piecewise linear approximations of the Hopf equation are presented in Section III. Dynamic and error analysis of the proposed models is considered in Section IV. Section $\mathrm{V}$ presents the circuit models and simulation results are evaluated in Section VI, where the paper is concluded in the last section.

\section{Hopf Resonator BASED 2-D COCHLEA}

Recent studies on the cochlea indicates its behavior as a selective amplifier transducing mechanical vibrations along the basilar membrane (BM) into neural activity. It is evident that the cochlea operates dynamically on or near to a Hopf bifurcation [29], [31], [32] based on which its extraordinary sensitivity and compression in amplitude response can be explained. These multidimensional biological resonators are too complicated to be represented by ordinary lumped components. As shown in [21], a VLSI-implemented cochlea, in comparison to the theoretical model, common simplifying assumptions are not able to preserve cochlear behavior [17]. Furthermore, couplings between resonators can significantly escalate complexity of the whole system. The Hopf equation based model adequately reproduces the main features of the cochlear response in simulations - thus, we select this model [3], [4], [13], [17]. The 2-D cochlear model in the fluid of the inner ear is shown in Fig. 1. This model includes a grid of two conductances, $G_{X}$ and $G_{Y}$, as proposed in [4]. Mathematically, the dynamics of each resonator can be described by a complex valued Hopf differential equation as follows [13], [33]:

$$
\dot{z}=z\left(\left(\mu+j \omega_{0}\right)-z^{2}\right)+v(t)
$$

where $z=x+j y$, which is the output signal, $\mu$ and $\omega_{0}$ are the control parameter (set point) and resonance frequency respectively and $v(t)$ is the external driving stimulus. The control parameter, $\mu$, is varied to obtain sub-critical, critical and unstable (limit cycle) solutions of (1). Table I demonstrates the regions of operation for a dynamical system governed by (1) [4]. The

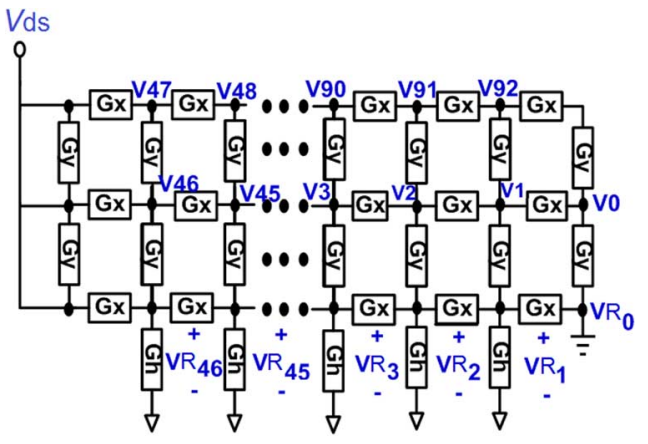

Fig. 1. The 2-D cochlea model includes a grid of two conductances, $G_{X}$ and $G_{Y}$, the applied driving stimulus to each resonator, $V_{R_{i}}$ and the conductance of the Hopf resonator, $G_{\mathrm{h}}[4]$.

TABLE I

REGIONS OF OPERATION

\begin{tabular}{c|c|c}
\hline Num. & $\mu$ & Region of operation \\
\hline 1 & $>0$ & Unstable, limit cycle \\
\hline 2 & $=0$ & Critical point, bifurcation (supercritical point) \\
\hline 3 & $<0$ & Sub-critical, stable equilibrium \\
\hline
\end{tabular}

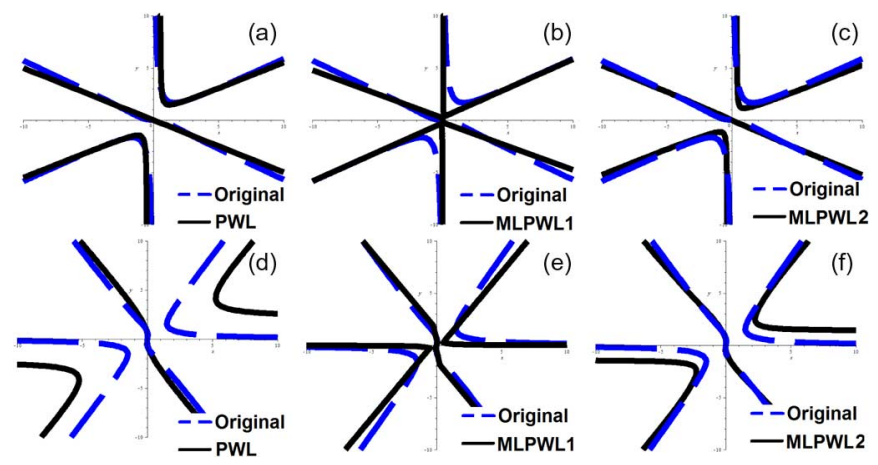

Fig. 2. The equilibrium $y-x$ locus of the proposed models fitted to the original model in $\omega_{0}=6 \mathrm{k} \cdot \mathrm{rad} / \mathrm{s}, \mu=-0.5$. (a) Real part equation of PWL model. (b) Real part equation of MLPWL1 model. (c) Real part equation of MLPWL2 model. (d) Imaginary part equation of PWL model. (e) Imaginary part equation of MLPWL1 model. (f) Imaginary part equation of MLPWL2 model.

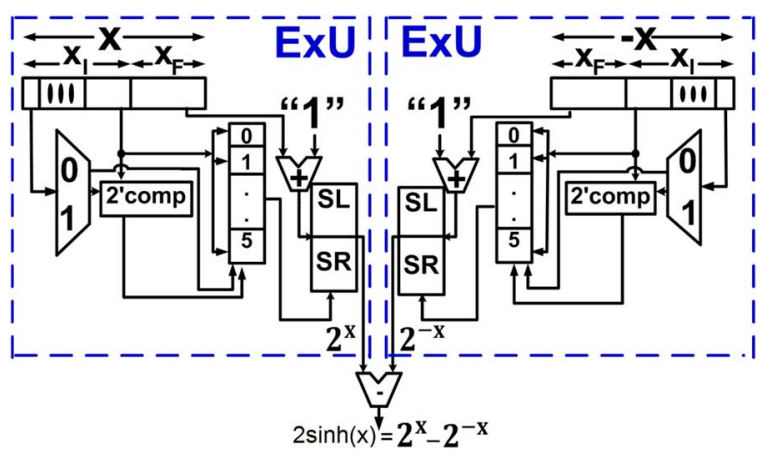

Fig. 3. Hyperbolic calculation unit (HCU) using: exponential unit (ExU), shiftto-left (SL), shift-to-right (SR), twos complement ( $2^{\prime}$ comp).

output signal is obtained from (1) can be decomposed into the real and imaginary parts to describe dynamic of the nonlinear active resonator as

$$
\left\{\begin{array}{l}
\dot{x}=\left(\mu-x^{2}+3 y^{2}\right) x-\omega_{0} y+v(t) \\
\dot{y}=\left(\mu-3 x^{2}+y^{2}\right) y+\omega_{0} x
\end{array}\right.
$$



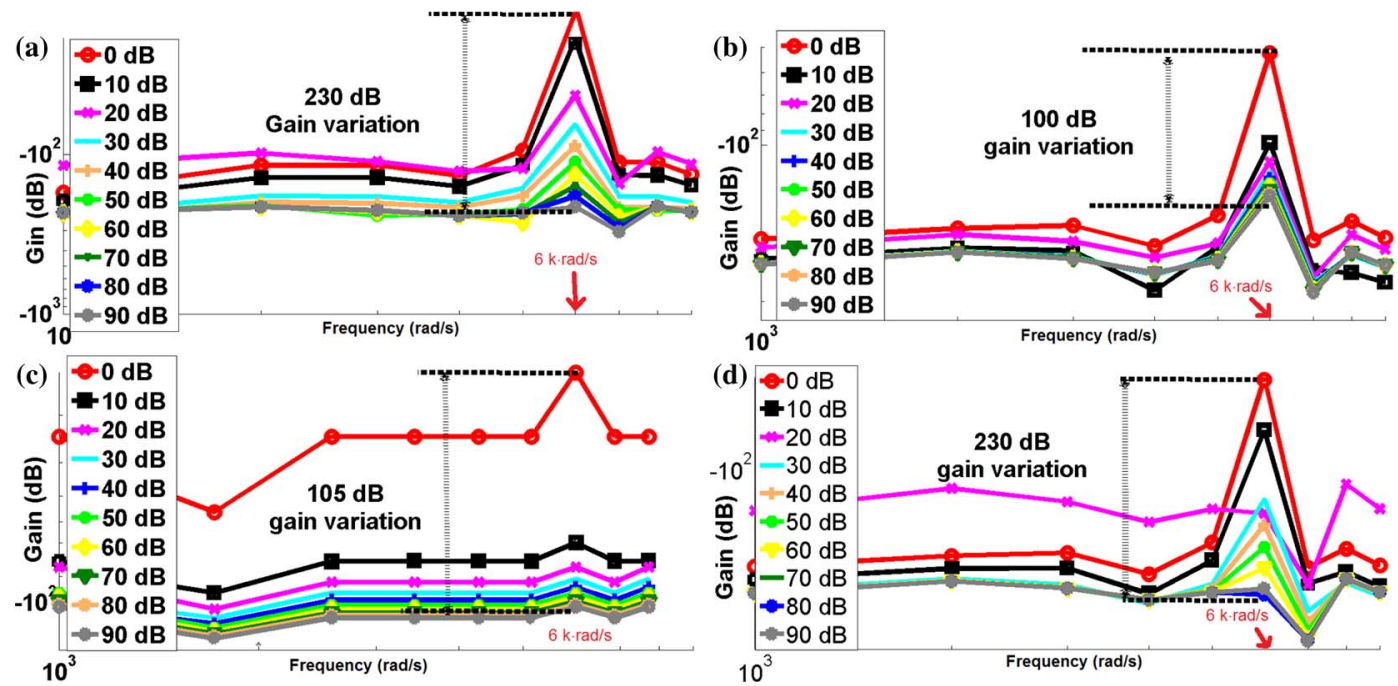

Fig. 4. The frequency responses of the original and proposed models where the characteristic frequency is $6 \mathrm{k} \cdot \mathrm{rad} / \mathrm{s}$. (a) Original model. (b) PWL model. (c) MLPWL1 model. (d) MLPWL2 model.

where $x$ and $y$ are real and imaginary parts of $z$. From a hardware implementation viewpoint, it should be noted that the nonlinear terms such as $x^{3}, y^{3}, x^{2} y$, and $y^{2} x$ need several multiplications, which is a costly functional unit for both digital and analog platforms. The next section proposes three models aimed at reducing the implementation cost.

\section{The PRoposed Models}

\section{A. Mathematical Approximation}

1) Piecewise Linear (PWL) Model: As previously mentioned, for an efficient hardware implementation, the nonlinear terms of $x^{3}, y^{3}, x^{2} y$, and $y^{2} x$ need to be replaced by computationally cost effective approximations. In the first modification, we propose to use $2|y|-|x|$ and $|y|-(3 / 2)|x|$ in (2) as follows:

$$
\text { PWL : }\left\{\begin{array}{l}
\dot{x}=(\mu+2|y|-|x|) x-\omega_{0} y+v(t) \\
\dot{y}=\left(\mu+|y|-\frac{3}{2}|x|\right) y+\omega_{0} x
\end{array}\right.
$$

As shown in Fig. 2(a) and Fig. 2(d), the proposed PWL model closely fits on the original equation.

2) Multiplierless Piecewise Linear Models (MLPWL1 and $M L P W L 2):$ Although the PWL model has a considerably lower implementation cost compared with the original model, requires two multipliers. Hence, for have a multiplierless implementation, we utilize $\operatorname{sign}(\cdot)$ and $\sinh (\cdot)$ functions that with our piecewise approximation can be implemented using simple shift and add operations:

$$
\begin{aligned}
& \text { MLPWL1 }:\left\{\begin{array}{l}
\dot{x}=(\mu+2|y|-|x|) \operatorname{sign}(x)-\omega_{0} y+v(t) \\
\dot{y}=\left(\mu+|y|-\frac{3}{2}|x|\right) \operatorname{sign}(y)+\omega_{0} \operatorname{sign}(x) .
\end{array}\right. \\
& \text { MLPWL2 }:\left\{\begin{array}{l}
\dot{x}=(\mu+2|y|-|x|) \sinh (x)-\omega_{0} y+v(t) \\
\dot{y}=8\left(\mu+|y|-\frac{3}{2}|x|\right) \sinh (y)+\omega_{0} x .
\end{array}\right.
\end{aligned}
$$

These models are fitted to the original model in Fig. 2(b), 2(c), 2(e), and 2(f). At circuit level, sign (.) can be implemented using basic full-wave rectifiers. Moreover, $\sinh (\cdot)$ is implementable using the presented approach in [34] based on a hyperbolic calculation unit (HCU) that is designed as shown in Fig. 3. The frequency responses of the original and proposed models are compared in Fig. 4 at $\omega_{0}=6 \mathrm{k} \cdot \mathrm{rad} / \mathrm{s}$. When the system is supercritical and it is driven at a characteristic frequency, the response is nonlinear with a high gain for small driving forces and smaller gain for larger driving forces. For other driving frequencies, the response is approximately linear for sufficiently small input [12], [26]. This significant feature is observed similarly in all proposed models.

\section{B. Dynamics and Phase Plane Trajectories}

To develop more rigorous justifications for the proposed models, the nullclines provide an insight into the level of similarity in dynamics compared to the original model. The nullclines of a variable are the clines where the time derivative is zero [35], [36]. Accordingly, considering $\dot{x}=0$ and $\dot{y}=0$, the nullclines are given by:

$$
\begin{array}{r}
\text { Original : }\left\{\begin{array}{l}
0=\left(\mu-x^{2}+3 y^{2}\right) x-\omega_{0} y+v(t) \\
0=\left(\mu-3 x^{2}+y^{2}\right) y+\omega_{0} x
\end{array}\right. \\
\text { PWL : }\left\{\begin{array}{l}
0=(\mu+2|y|-|x|) x-\omega_{0} y+v(t) \\
0=\left(\mu+|y|-\frac{3}{2}|x|\right) y+\omega_{0} x
\end{array}\right. \\
\text { MLPWL1: }\left\{\begin{array}{l}
0=(\mu+2|y|-|x|) \operatorname{sign}(x)-\omega_{0} y+v(t) \\
0=\left(\mu+|y|-\frac{3}{2}|x|\right) \operatorname{sign}(y)+\omega_{0} \operatorname{sign}(x)
\end{array}\right. \\
\text { MLPWL2 : }\left\{\begin{array}{l}
0=(\mu+2|y|-|x|) \sinh (x)-\omega_{0} y+v(t) \\
0=8\left(\mu+|y|-\frac{3}{2}|x|\right) \sinh (y)+\omega_{0} x
\end{array}\right.
\end{array}
$$

where $v(t)=0$ and there are fixed points with coordinates for each model. To determine the type of equilibrium points, eigenvalues of the system are calculated as

$$
J(x, y)=J\left(x_{\mathrm{eq}}, y_{\mathrm{eq}}\right)=\left[\begin{array}{ll}
A & B \\
C & D
\end{array}\right]
$$

where $A, B, C$, and $D$ are the coefficients of the Jacobian matrix as presented in Table II. This analysis shows that behavior of the proposed models (specially MLPWL2 and PWL models) without input are quite similar to the original model as shown in Fig. 5 [1-(a), 1-(b), 1-(c), and 1-(d)]. In addition, it can be seen that the origin is a globally stable equilibrium point of the 
TABLE II

Equilibrium Point Data, Jacobian, Eigenvalues, and Eigenvectors of Original and Proposed Models IN $\omega_{0}=6 \mathrm{k} \cdot \mathrm{rad} / \mathrm{s}$. Abbreviations: Equ. Point $=$ Equilibrium Point, S. So. = Spiral Source, S. Si. = Spiral SinK, N. So. = Nodal Source, N. Si. = Nodal SinK, S. P. = SAdDle POINT

\begin{tabular}{|c|c|c|c|c|c|c|c|c|c|c|c|c|}
\hline \multirow[t]{2}{*}{ input } & \multicolumn{6}{|c|}{ Original model } & \multicolumn{6}{|c|}{ PWL model } \\
\hline & Equ. point & type & A & B & $\mathrm{C}$ & $\mathrm{D}$ & Equ. point & type & A & B & $\mathrm{C}$ & $\mathrm{D}$ \\
\hline 0 & $(54.77,54.77)$ & S. So. & 1 & 12000 & -11999 & 1 & $(10725,7445)$ & S. Si. & -6560 & 15450 & -5168 & -1197 \\
\hline 0 & $(-54.77,-54.77)$ & S. So. & 0.98 & 11999 & -11999 & 0.98 & $(0,0)$ & S. Si. & -0.5 & -6000 & 6000 & -0.5 \\
\hline 0 & $(-54.77,-54.77)$ & S. Si. & -1.17 & 12000 & -12000 & -1.17 & $(-10725,-7445)$ & S. Si. & -6560 & 15450 & -5168 & -1197 \\
\hline 10 & $(0,0.001)$ & S. Si. & -0.499 & -6000 & 6000 & -0.499 & $(10725,7445)$ & S. Si. & -6560 & 15450 & -5168 & -1197 \\
\hline 10 & $(-54.77,-54.77)$ & S. So. & 1.27 & 12000 & -12000 & 1.2736 & $(0,0.001)$ & S. Si. & -0.4966 & -6000 & 5999 & -0.4966 \\
\hline 10 & $(54.76,54.77)$ & S. So. & 0.83 & 11999 & -11999 & 0.83057 & $(-10725,-7445)$ & S. Si. & -6560 & 15450 & -5168 & -1197 \\
\hline \multirow[t]{2}{*}{ input } & \multicolumn{6}{|c|}{ MLPWL1 model } & \multicolumn{6}{|c|}{ MLPWL2 model } \\
\hline & Equ. point & type & A & B & $\mathrm{C}$ & D & Equ. point & type & A & B & $\mathrm{C}$ & D \\
\hline 0 & - & - & - & - & - & - & $(-49.29,-424.5)$ & N. So. & 1.02 & 2.56 & -1.47 & 3.457 \\
\hline 0 & - & - & - & - & - & - & $(-91.49,249)$ & N. So. & 1.1 & -5.45 & 9.08 & 6.8 \\
\hline 0 & - & - & - & - & - & - & $(-217,-73.74)$ & N. Si. & -1.39 & 3.89 & -6.4 & -1.07 \\
\hline 10 & - & - & - & - & - & - & $(-8.73,-424.58)$ & N. So. & 260.5 & 211 & -1.49 & 1.09 \\
\hline 10 & - & - & - & - & - & - & $(-44.35,-73.73)$ & N. So. & 9.35 & 1.84 & -6.35 & 3.26 \\
\hline 10 & - & - & - & - & - & - & $(-49.3,249.1)$ & N. So. & 5.81 & -2.59 & 9.3 & 1.08 \\
\hline
\end{tabular}
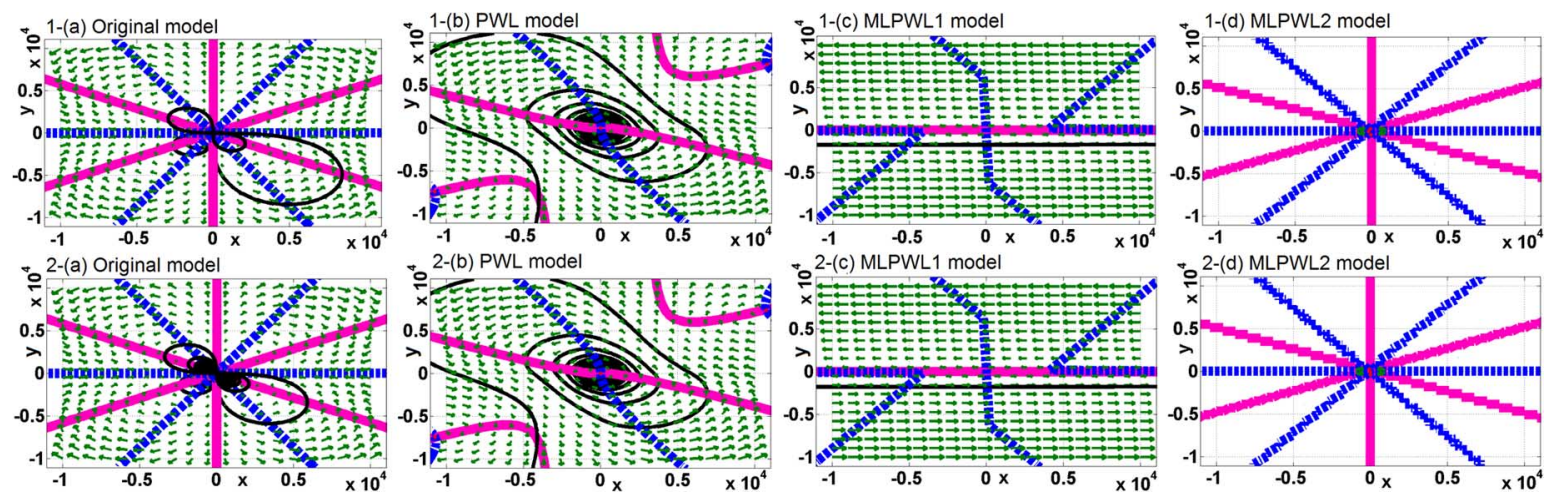

Fig. 5. X-Y curve and phase portrait of original, PWL, MLPWL1, and MLPWL2 models and nullclines of these systems with increasing the current for four states of (1) to (2) with these conditions $\mu=-0.5$ (stable region) and $\omega_{0}=6 \mathrm{k} \cdot \mathrm{rad} / \mathrm{s}$. This figure shows how the increasing of the input signal $(v)$ changes the state of the nullclines, redound to bifurcation, and finally trajectories of these equations. The blue dashed lines represent Y-nullclinces, pink lines represent $\mathrm{X}$-nullclinces, black lines represent the trajectory at green vector fields.

TABLE III

Degree of Error Between the Modified Formula And Original Model With Time - Step $=0.0001$ AND $\omega_{0}=6 \mathrm{k} \cdot \mathrm{rad} / \mathrm{s}$

\begin{tabular}{c|c|c|c|c|c|c|c|c}
\hline Num. & Intensity & Freq. & \multicolumn{2}{|c|}{ PWL } & \multicolumn{2}{c|}{ MLPWL1 } & \multicolumn{2}{c}{ MLPWL2 } \\
& $(\mathrm{dB})$ & $\mathrm{k} \cdot \mathrm{rad} / \mathrm{s}$ & RMSE & NRMSE & RMSE & NRMSE & RMSE & NRMSE \\
\hline 1 & -2 & 6 & $1.2410 \times 10^{-7}$ & 0.1124 & $1.6039 \times 10^{-5}$ & 14.5217 & $3.9056 \times 10^{-7}$ & 0.3538 \\
\hline 2 & -8 & 6 & $1.3476 \times 10^{-7}$ & 0.1181 & $1.3983 \times 10^{-5}$ & 12.2519 & $3.0618 \times 10^{-7}$ & 0.2684 \\
\hline 3 & -20 & 6 & $1.4278 \times 10^{-7}$ & 0.1220 & $5.0396 \times 10^{-6}$ & 4.3074 & $2.4668 \times 10^{-7}$ & 0.2109 \\
\hline 4 & -26 & 6 & $1.4413 \times 10^{-7}$ & 0.1227 & $1.9750 \times 10^{-5}$ & 16.8105 & $6.6268 \times 10^{-7}$ & 0.5644 \\
\hline Mean error & - & - & $1.3644 \times 10^{-7}$ & 0.1188 & $1.3717 \times 10^{-5}$ & 11.9728 & $4.0152 \times 10^{-7}$ & 0.3493 \\
\hline
\end{tabular}

total system. Thus, for all values of $v(t)$, the models are globally stable except for the MLPWL1 model for some driving stimuli. Thus, in similar accordance to the original model, there are stable states in the proposed models that are shown in Fig. 5 [2-(a), 2-(b), 2-(c) and 2-(d)]. The gain variations of the original, PWL, MLPWL1, and MLPWL2 models are $230 \mathrm{~dB}, 100$ $\mathrm{dB}, 105 \mathrm{~dB}$, and $230 \mathrm{~dB}$ respectively, as shown in Fig. 4.

\section{Error Analysis}

Root mean square error (RMSE) is used to analyze precision of the proposed modified equation as an approximation for the original differential equation. We define RMSE and normalized root mean square error (NRMSE) as

$$
\begin{aligned}
\operatorname{RMSE}\left(V_{\mathrm{pm}}, V_{\text {original }}\right) & =\sqrt{\frac{\sum_{1}^{n}\left(V_{\mathrm{pm}}-V_{\text {original }}\right)^{2}}{n} .} \\
\mathrm{NRMSE} & =\frac{\mathrm{RMSE}}{V_{\max }-V_{\min }}
\end{aligned}
$$

where $V_{\text {original }}$ is the original value and $V_{\mathrm{pm}}$ is the approximated value that are presented in Table III. Using these measures, errors for each input intensity level with different frequency is computed for a time step of 0.0001. Single tones in sinusoidal form are used to compute the errors. Corresponding results, given in Table III, indicate an acceptable precision for the 


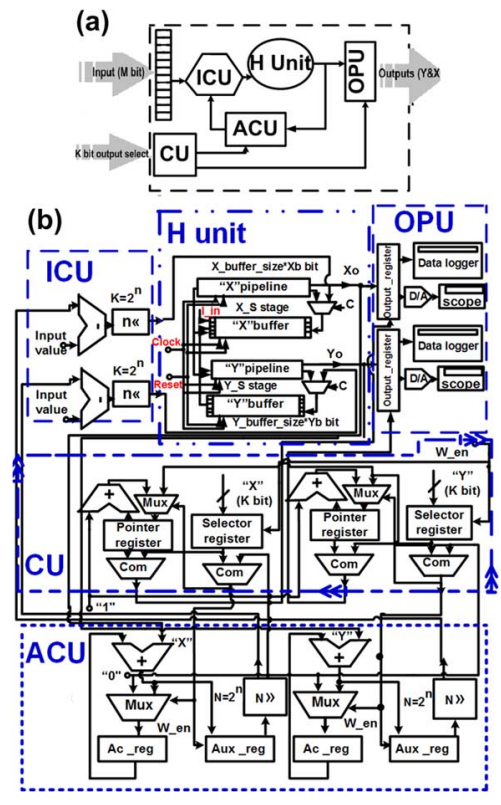

Fig. 6. The proposed system architecture. (a) System block diagram. (b) Hopf resonator array block diagram. Abbreviations: Hopf unit (H Unit), input computing unit (ICU), output provider unit (OPU), control unit (CU), average computing unit (ACU)

proposed methods. The mean normalized root mean square errors (NRMSEs) of the PWL, MLPWL1, and MLPWL2 models are $0.11 \%, 11.97 \%$, and $0.34 \%$, respectively, as compared to the original cochlea model.

\section{Design AND HaRdware IMPLEMENTATION}

As shown in Fig. 6, the proposed system architecture for the MLPWL2 model includes a system block diagram and a Hopf resonator array block diagram. This architecture is expanded in detail as following subsections.

\section{A. Equation Discretization}

Based on (2)-(5), each model includes two blocks to compute $\dot{x}$ and $\dot{y}$. As the first implementation step, it is essential to discretize the related equations. In this study, we have applied the Euler method.

\section{B. Hopf Unit}

As shown in Fig. 7, this unit is a digital multiplierless implementation of the Hopf resonator model as in (5). This unit includes $X_{\text {pipeline }}, Y_{\text {pipeline }}, X_{\text {buffer }}$, and $Y_{\text {buffer }}$. Here, $X_{\text {pipeline }}$, $Y_{\text {pipeline }}$ are $x$ and $y$ in (5) respectively. These variables are implemented in a pipeline structures with $X_{\text {stage }}$ and $Y_{\text {stage }}$ stages, where $X_{\text {buffer }}$ and $Y_{\text {buffer }}$ are the buffer registers of the $x$ and $y$ values and $X_{\text {buffer-size }}$ and $Y_{\text {buffer-size }}$ are the size of $x$ and $y$ states. With every rising edge of the clock, these buffers are shifted to obtain new values. The bit number can be calculated, according to usage and precision. Based on the variable equations, to create repetitive states, buffer outputs are applied to the related arithmetic units. None of these arithmetic modules are in pipeline state, which means any arithmetic module requires a register after each arithmetic state in order to save data.

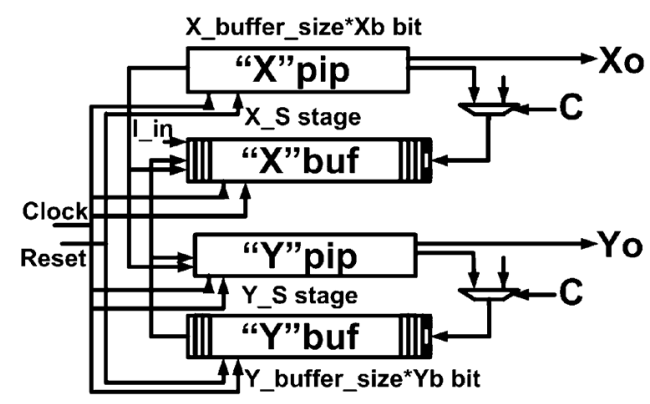

Fig. 7. General overview of $\mathrm{H}$ unit that is a digital multiplierless implementation of the Hopf resonator model. This unit includes $X_{\text {pipeline }}, Y_{\text {pipeline }}$, $X_{\text {buffer }}$ and $Y_{\text {buffer }}$. Here, $X_{\text {pipeline }}, Y_{\text {pipeline }}$ are presented $x$ and $y$ in (5), respectively. These variables are performed in pipeline structures with $X_{\text {stage }}$ and $Y_{\text {stage }}$ stages, where $X_{\text {buffer }}$ and $Y_{\text {buffer }}$ are the buffer registers for the $x$ and $y$ values and $X_{\text {buffer-size }}$ and $Y_{\text {buffer-size }}$ are the size of $x$ and $y$ states. (pip $=$ pipeline, buf $=$ buffer $)$

Accordingly, the following conditions must be satisfied:

$$
\left\{\begin{array}{l}
N=x_{\text {buffer }- \text { size }}+x_{\text {stage }}=y_{\text {buffer }- \text { size }}+y_{\text {stage }} \\
x_{\text {buffer-size }}=y_{\text {buffer }- \text { size }} \\
x_{\text {stage }}=y_{\text {stage }}
\end{array}\right.
$$

where $N$ is the number of Hopf resonators. The delay of each stage is scheduled in order to attain synchronization of the equations. In each clock step, the average values of $x$ and $y$ are calculated and in the next clock step are applied. This guarantees that the final Hopf resonator provides output with an appropriate timing. Then first resonator is updated to a new value on the next rising clock edge. In this condition the input is applied to the resonator at the latest step of the pipeline stages. This process omits the average delay of the computing that is updated in the next time sample for applying to a resonator. For an improved comparison between original, PWL, MLPWL1, and MLPWL2 models, the scheduling diagrams (data flow graph with scheduling control steps) based on (3), (4), and (5) are drawn in Fig. 8.

\section{Control Unit}

The control unit (CU) is responsible for two major tasks: 1) Providing suitable signal for the output provider unit in order to select a suitable Hopf resonator that is selected by the K-bit input of the user defined number for applying. 2) Providing suitable signal for the average computing unit in order to calculate the average value of resonator's output. The architecture of the control unit is presented in Fig. 9. The CU is equipped with a counter that acts as a pointer and obtains the number of the Hopf resonator. For the rising edge of the clock pulse, this counter is increased by one; and when the counter reaches $N$, a reset is performed. The comparator checks the value of the counter register as a pointer-register and when the counter is $N$, the time is reset. The reset instruction points to the first resonator and sends it to the average computing unit as an instruction. The comparator, compares the value of the pointer-register with a selected Hopf resonator that is inserted in the selector-register and sends a suitable instruction to the output provider unit (OPU). The selector-register may also be updated by the enable signal as write-enable. 


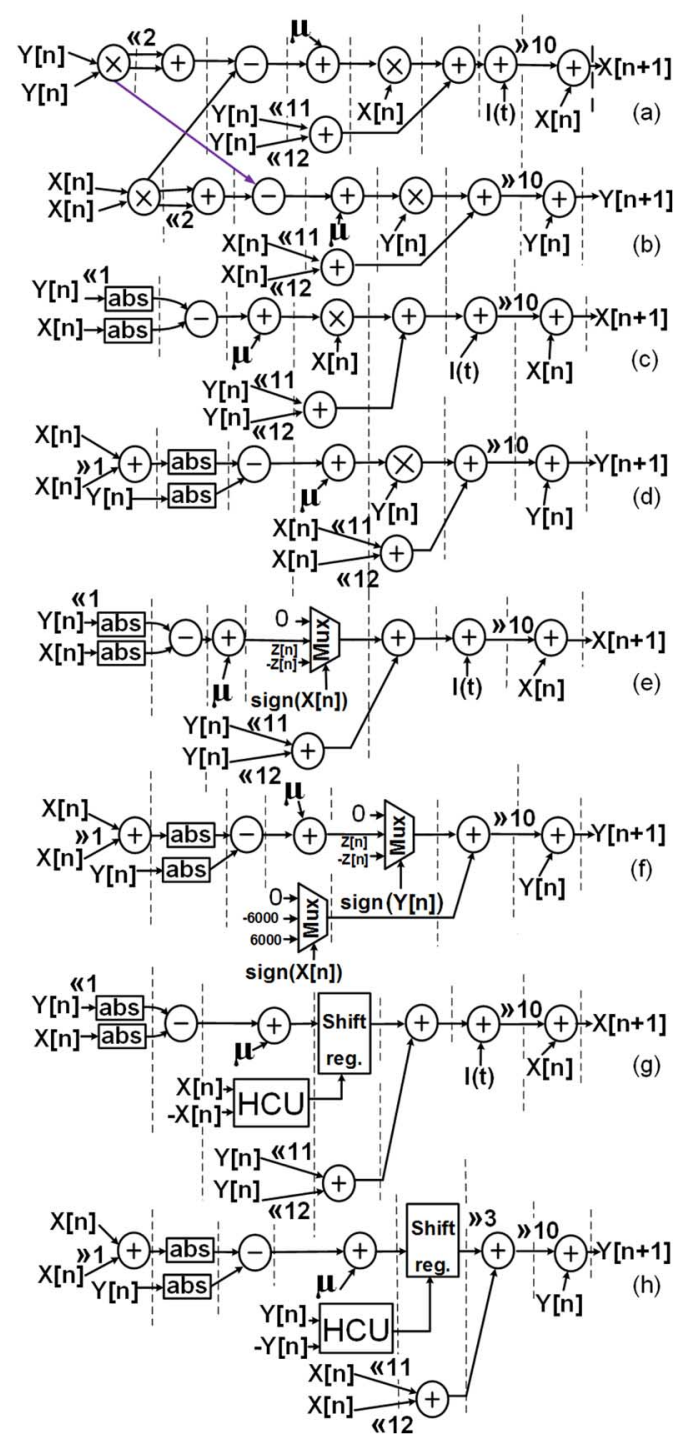

Fig. 8. Arithmetic pipelines at $\omega_{0}=6 \mathrm{k} \cdot \mathrm{rad} / \mathrm{s}$. (a) $x$ pipeline in original model. (b) $y$ pipeline in original model. (c) $x$ pipeline in PWL model. (d) $y$ pipeline in PWL model. (e) $x$ pipeline in MLPWL1 model. (f) $y$ pipeline in MLPWL1 model. (g) $x$ pipeline in MLPWL2 model. (h) $y$ pipeline in MLPWL2 model.

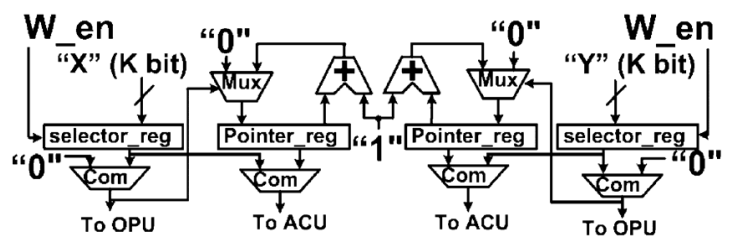

Fig. 9. The control unit $(\mathrm{CU})$ of the proposed model. Abbreviations: comparator (Com), average computing unit (ACU), output provider unit (OPU), write enable (W_en).

\section{Average Computing Unit}

Average computing unit (ACU) computes $x$ and $y$ state variables. The architecture of ACU is presented in Fig. 10. The ACU performs the task by reading the accumulator-register with a serial accumulator repetitively and accumulates it with $x$ and $y$ variables from the $\mathrm{H}$ unit and the outcome is returned to the register on every clock pulse. The accumulator-register stores $N-1$ numbers of $x$ and $y$ input variables in the $(N$ $-1)$ th clock pulse after the latest reset. Therefore, the adder's

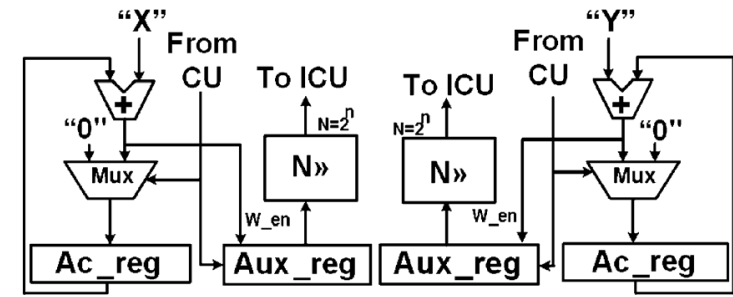

Fig. 10. General structure of the average computation unit (ACU). Abbreviations: Accumulator_register (Ac_reg), Auxiliary_register (Ac_reg), write enable (W_en).

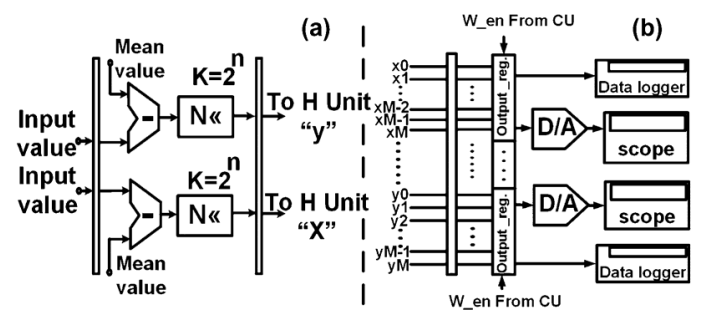

Fig. 11. General overview of input computation unit and output provider unit (a) Input computation unit. (b) Output provider unit.

output is the final addition of $N$ variables of $x$ and $y$ at the mentioned clock pulses. This amount must be saved in an auxiliary-register after $N$ clock pulses in order to apply a Hopf resonator model in the next time sample. Value of auxiliary-register must be divided by $N$ average values which are sent to the input computing unit. We have selected $N$ as a power of 2 for replacing multiplication operation by shift operation.

\section{E. Input Computing Unit}

The input computing unit (ICU), is designed as a structure, shown in Fig. 11(a), that caches the input, $x$ and $y$ variables averages from the input port and ACU, and then computes the difference. Afterward, it multiplies the result by constant $K$. The ICU must perform this operation without any delay. After computing new average which is produced by average computing unit, it should give the updated input to the Hopf resonators immediately. Therefore, this unit is designed via combination of gates and does not include any register. We should notice that the implementation complexity of $K$ constant multiplier can be substantially decreased by selecting suitable value for $K$. If suitable value of $K$ is chosen, one may implement it with pairs of accumulators and shift logic operations. In this paper, we select $K$ in such a way that multiplication can be replaced by simple shift operation.

\section{F. Output Provider Unit}

The output provider unit (OPU), shown in Fig. 11(b), saves $x$ and $y$ as the variables of the Hopf resonator models in the output-register in $N$ clock pulses. The control unit provides writing activation for this register in order to save valid $x$ and $y$. The OPU also transforms saved digital numbers in the output-register to analog signal by digital analog converter (DAC).

For a 2-D artificial cochlea implementation, according to the mentioned model, it is sufficient to obtain a relationship between input signal as a total input driving stimulus, which is applied to 


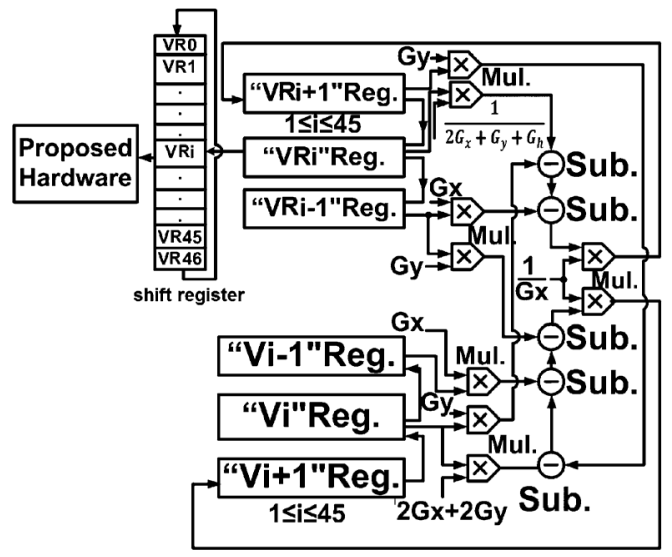

Fig. 12. The active nonlinear artificial cochlea is implemented using a single resonator. Abbreviations: Subtractor (Sub), Multiplier (Mull).

the resonator networks, and the corresponding value that is applied to each resonator. According to Fig. 1, the relationship between the total input driving stimulus $\left(V_{\mathrm{ds}}\right)$ and the input signal, which is applied to each resonator at the $i$ th resonator, $V_{R_{i}}, V_{i}$, and $V_{\mathrm{ds}}$ respectively are given by the following equations:

$$
\left\{\begin{array}{l}
V_{R_{i}}=\frac{G_{\mathrm{x}}\left(V_{R_{i-1}}+V_{R_{i+1}}\right)+V_{i} G_{\mathrm{y}}}{G_{\mathrm{h}}+2 G_{\mathrm{x}}+G_{\mathrm{y}}} \\
V_{i}=\frac{\left(V_{R_{i-1}}+V_{R_{i+1}}\right) G_{\mathrm{y}}+\left(V_{i-1}+V_{i+1}\right) G_{\mathrm{x}}}{2 G_{x}+2 G y} \\
1 \leq i \leq 45
\end{array}\right.
$$

and

$$
\left\{\begin{array}{l}
V_{i}=\frac{\left(V_{93-i}\right) G_{\mathrm{y}}+\left(V_{i-1}+V_{i+1}\right) G_{\mathrm{x}}}{2 G_{\mathrm{x}}+G_{\mathrm{y}}} \\
48 \leq i \leq 91
\end{array}\right.
$$

for $i=0, i=46, i=47$, and $i=92$ it can be written that:

$$
\left\{\begin{array}{l}
V_{0}=\frac{V_{92}\left(G_{\mathrm{x}}+G_{\mathrm{y}}\right)+\left(V_{1}\right) G_{\mathrm{x}}}{2 G_{\mathrm{x}}+2 G_{\mathrm{y}}} \\
V_{\mathrm{R}_{46}}=\frac{G_{\mathrm{x}}\left(V_{\mathrm{R}_{45}}+V_{\mathrm{ds}}\right)+V_{46} G_{\mathrm{y}}}{G_{\mathrm{h}}+2 G_{\mathrm{x}}+G_{\mathrm{y}}} \\
V_{46}=\frac{\left(V_{\mathrm{R}_{45}}+V_{\mathrm{ds}}\right) G_{\mathrm{y}}+\left(V_{45}+V_{\mathrm{ds}}\right) G_{\mathrm{x}}}{2 G_{x}+2 G_{\mathrm{y}}} \\
V_{47}=\frac{\left(V_{46}\right) G_{\mathrm{y}}+\left(V_{\mathrm{x}}+V_{\mathrm{ds}}\right) G_{\mathrm{x}}}{2 G_{x}+G_{\mathrm{y}}} \\
V_{92}=\frac{\left(V_{0}+V_{91}\right) G_{\mathrm{x}}+\left(V_{0}+V_{1}\right) G_{\mathrm{y}}}{2 G_{\mathrm{x}}+2 G_{\mathrm{y}}}
\end{array}\right.
$$

where $V_{R_{i}}$ is the applied driving stimulus to each resonator, $G_{\mathrm{h}}$ is the conductance of the Hopf resonator, $V_{0}, V_{R_{46}}, V_{46}, V_{47}$, and $V_{92}$ are boundary condition (12) and (13). Interestingly, the proposed 2-D cochlea can be implemented using a single resonator that we proposed-instead of 46 (or 12) resonators as in previous studies [4]. This implementation consists of: I) Nine multipliers, II) Five subtractors, III) Several registers for constant coefficients $\left(G_{\mathrm{x}}, G_{\mathrm{y}}, 1 / G_{\mathrm{x}}, 1 /\left(2 G_{\mathrm{x}}+G_{\mathrm{y}}+G_{\mathrm{h}}\right)\right.$ and $\left.2 G_{\mathrm{x}}+2 G_{\mathrm{y}}\right)$, generated driving stimulus amounts $\left(V_{R_{i+1}}, V_{R_{i}}\right.$ and $\left.V_{R_{i-1}}\right)$ and the voltages of the nodes $\left(V_{i+1}, V_{i}\right.$ and $\left.V_{i-1}\right)$ in which $i$ varies from 1 to 45, IV) A 47-bit shift register is used to save all $V_{R_{i}}$ values generated in each step. This implementation is presented in Fig. 12.

\section{Simulation AND IMPlementation Results}

The resonator behavior is described using synthesizable VHDL code. In these simulations word-length of $x$ and $y$ are 32 bits which are used in a fixed point calculation with 18 bits for integer values and 14 bits for fractional values. Resonator

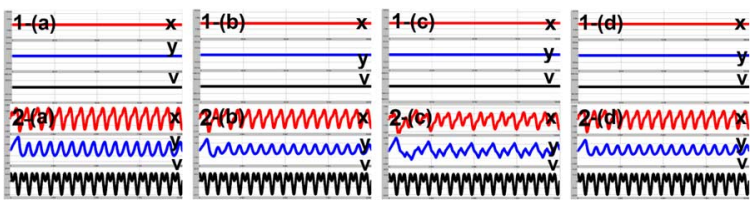

Fig. 13. Output of the Original and proposed models. (a) Original model. (b) PWL model. (c) MLPWL1 model. (d) MLPWL2 model. (1) $V(t)=0$ volt. (2) $V(t)=10 \sin (10 \pi t)+10 \cos (20 \pi t)$ volt.

TABLE IV

The Number of Minimum Resources IN THE SCHEDUling of $x$ AND $y$

\begin{tabular}{c|c|c|c|c|c}
\hline Resources & Output & $\begin{array}{c}\text { Original } \\
\text { Model }\end{array}$ & $\begin{array}{c}\text { PWL } \\
\text { Model }\end{array}$ & $\begin{array}{c}\text { MLPWL1 } \\
\text { Model }\end{array}$ & $\begin{array}{c}\text { MLPWL2 } \\
\text { Model }\end{array}$ \\
\hline Adder & $x$ & 7 & 6 & 6 & 9 \\
\hline Multiplier & $x$ & 3 & 1 & - & - \\
\hline Multiplexer & $x$ & - & - & 1 & - \\
\hline Adder & $y$ & 6 & 6 & 6 & 9 \\
\hline Multiplier & $y$ & 3 & 1 & - & - \\
\hline Multiplexer & $y$ & - & - & 2 & - \\
\hline
\end{tabular}

parameters are set as $\mu=-0.5, \omega_{0}=6 \mathrm{k} \cdot \mathrm{rad} / \mathrm{s}$ and initial values are $x_{0}=1 / 1024$ and $y_{0}=1 / 1024$. The device utilization for implementation of a single resonator based on the Hopf equations and the proposed models are summarized in Table $\mathrm{V}$.

For a fair comparison between the original model and the proposed models, we applied the same input signals as driving stimulus to all designs. Results are shown in Fig. 13. Response of the proposed models have acceptable similarity to the original model. Single tone, two tone, and DC signals are considered as inputs. One of the essential conditions of a stable systems is its zero response to the zero input signal. To show stability of the original model and the proposed models, driving stimulus $v(t)$ is set to zero. The necessary resources for a single resonator are considered for both PWL and MLPWL models in a pipeline configuration with implementation results as presented in Table IV. Circuits are implemented on a XILINX Virtex-II Pro development board. Fig. 14 shows oscilloscope photographs of PWL, MLPWL1, and MLPWL2 models implementation. As observable, the MLPWL1 model has lower cost but higher error than PWL. However, MLPWL1 has acceptable precision, but MLPWL2 model has excellent accuracy and lower cost in comparison with other models.

\section{CONCLUSION}

A set of piecewise linear active cochlear models, which closely follows the biological cochlea targeting low cost digital implementation have been presented. Three piecewise linear models are proposed namely PWL, MLPWL1, and MLPW2, which have the same characteristics of the Hopf resonators for active nonlinear 2-D artificial cochlea implementation. The system level simulation results demonstrate that the MLPWL and PWL models have sensitivity to both amplitude and frequency of the input signals. Accordingly, a compact structure of the models to implement 2-D artificial cochlea implementation has been developed. The proposed models were investigated, in terms of digital implementation feasibility and costs, targeting large scale low cost hardware implementations. Hardware synthesis and physical implementations on FPGA show that the proposed models can reproduce precise active cochlea 
TABLE V

HARDWARE IMPLEMENTATION COST FOR DifFERENT MODELS

\begin{tabular}{|c|c|c|c|c|c|c|c|c|c|}
\hline Model & FFs & FFs. $\%$ & 4- LUTs & 4- LUTs\% & Add/sub & MULT18 $\times 18 s$ & MULT18 $\times 18 s \%$ & Min. period & Max. Freq. \\
\hline Original & 560 & 2 & 781 & 2 & 10 & 16 & 11 & $3.489 \mathrm{~ns}$ & $286.029 \mathrm{MHz}$ \\
\hline PWL & 497 & 1 & 755 & 2 & 14 & 8 & 5 & $3.182 \mathrm{~ns}$ & $314.175 \mathrm{MHz}$ \\
\hline MLPWL1 & 400 & 1 & 451 & 2 & 16 & 0 & 0 & $2.704 \mathrm{~ns}$ & $339.795 \mathrm{MHz}$ \\
\hline MLPWL2 & 434 & 1 & 481 & 2 & 21 & 0 & 0 & $3.084 \mathrm{~ns}$ & $324.219 \mathrm{MHz}$ \\
\hline
\end{tabular}

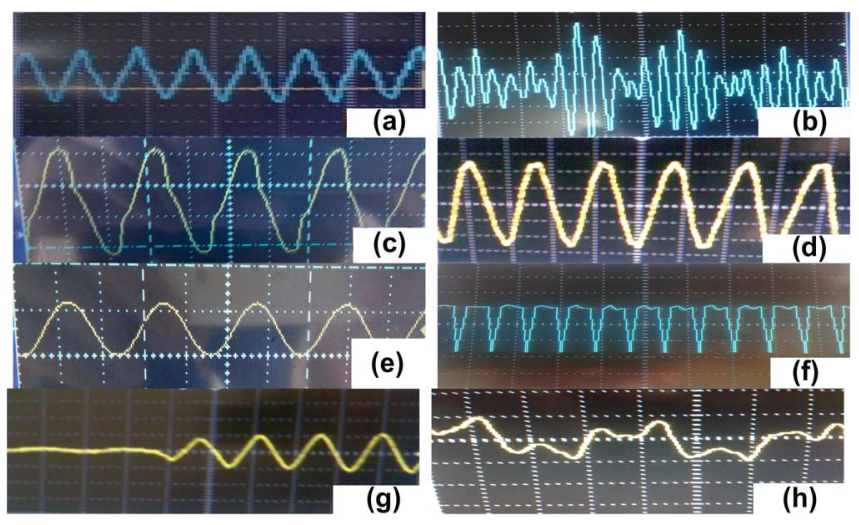

Fig. 14. Output of the MLPWL2 model implemented on XILINX Virtex-II Pro XC2VP30. (a) Single tone (intensity $=10$ volt, $F=60 \mathrm{rad} / \mathrm{s}$ ). (b) Two tone. (c) Single tone (intensity $=10$ volt, $F=10 \mathrm{rad} / \mathrm{s}$ ). (d) Single tone (intensity $=15$ volt, $F=10 \mathrm{rad} / \mathrm{s}$. (e) Single tone (intensity $=1$ volt, $F=10 \mathrm{rad} / \mathrm{s}$ ). (f) Two tone. (g) Various intensity ( $1 \mathrm{mV}$ to 3 volt) and $F$ $=10 \mathrm{rad} / \mathrm{s}$. (h) Two tone. The horizontal axis denotes time, and the vertical axis shows voltage.

behaviors with higher performance and considerably lower computation costs in comparison with the original model. The results indicate that the MLPWL1 model has lower computation, precision and hardware cost, while the PWL model has a higher precision and dynamically tracks similar to the original model. On the other hand, advantage of the MLPWL2 model is that it outperforms the others in accuracy, dynamically tracking the original model and is not as costly as the PWL model. Also dynamic behavior of the Hopf resonator has been tested for all models and the results indicate a good performance of proposed models.

\section{REFERENCES}

[1] J. P. Keener and J. Sneyd, Mathematical Physiology. New York: Springer, 1998, vol. 8.

[2] D. M. Rasetshwane, M. P. Gorga, and S. T. Neely, "Signal-processing strategy for restoration of cross-channel suppression in hearing-impaired listeners," IEEE Trans. Biomed. Eng., vol. 61, no. 1, pp. 64-75, Jan. 2014

[3] S. P. Bacon, R. R. Fay, and A. N. Popper, Compression: From Cochlea to Cochlear Implants. New York: Springer, 2004.

[4] T. J. Hamilton, J. Tapson, C. Jin, and A. van Schaik, "Analogue VLSI implementations of two dimensional, nonlinear, active cochlea models," in Proc. IEEE Biomed. Circuits Syst. Conf. (BioCAS), 2008, pp. $153-156$.

[5] M. P. Leong, C. T. Jin, and P. H. Leong, "An FPGA-based electronic cochlea," EURASIP J. Appl. Signal Process., vol. 7, pp. 629-638, 2003.

[6] S. Wang, T. J. Koickal, A. Hamilton, R. Cheung, and L. S. Smith, "A bio-realistic analog CMOS cochlea filter with high tunability and ultrasteep roll-off," IEEE Trans. Biomed. Circuits Syst., Jul. 2014, 10.1109/ TBCAS.2014.2328321.

[7] T. Gold, "Hearing. II. The physical basis of the action of the cochlea," Proc. Roy. Soc. London B, Biol. Sci., vol. 135, no. 881, pp. 492-498, 1948.

[8] R. F. Lyon and C. Mead, "An analog electronic cochlea," IEEE Trans. Acoust., Speech, Signal Process., vol. 36, no. 7, pp. 1119-1134, 1988.
[9] Y. Choe, M. O. Magnasco, and A. J. Hudspeth, "A model for amplification of hair-bundle motion by cyclical binding of $\mathrm{Ca}^{2+}$ to mechanoelectrical-transduction channels," Proc. Natl. Acad. Sci., vol. 95, no. 26, pp. 15321-15326, 1998.

[10] W. E. Brownell, C. R. Bader, D. Bertrand, and Y. De Ribaupierre, "Evoked mechanical responses of isolated cochlear outer hair cells," Science, vol. 227, no. 4683, pp. 194-196, 1985.

[11] M. A. Ruggero, L. Robles, and N. C. Rich, "Two-tone suppression in the basilar membrane of the cochlea: Mechanical basis of auditory-nerve rate suppression," J. Neurophysiol., vol. 68, pp. 1087-1087, 1992.

[12] M. B. Sachs and N. Y. Kiang, "Two tone inhibition in auditory nerve fibers," J. Acoust. Soc. Amer., vol. 43, no. 5, pp. 1120-1128, 1968

[13] T. J. Hamilton, J. Tapson, M. Rapson, C. Jin, and A. van Schaik, "Understanding the mathematics of hearing using electronic circuits," ANZIAM J., vol. 51, pp. C300-C315, 2010.

[14] J. P. Lazzaro and C. Mead, "A silicon model of auditory localization," Neural Comput., vol. 1, no. 1, pp. 47-57, 1989.

[15] J. P. Lazzaro and C. Mead, "Silicon modeling of pitch perception," Proc. Natl. Acad. Sci., vol. 86, no. 23, pp. 9597-9601, 1989.

[16] J. P. Lazzaro, J. Wawrzynek, and A. Kramer, "Systems technologies for silicon auditory models," IEEE Micro, vol. 14, no. 3, pp. 7-15, 1994.

[17] A. van Schaik and R. Meddis, "Analog very large-scale integrated (VLSI) implementation of a model of amplitudemodulation sensitivity in the auditory brainstem," J. Acoust. Soc. Amer., vol. 105, no. 2, pp. 811-821, 1999.

[18] C. A. Mead, X. Arreguit, and J. P. Lazzaro, "Analog VLSI model of binaural hearing," IEEE Trans. Neural Netw., vol. 2, no. 2, pp. 230-236, 1991.

[19] J. P. Lazzaro, J. Wawrzynek, and R. P. Lippmann, "Micro power analog circuit implementation of hidden Markov model state decoding," IEEE J. Solid-State Circuits, vol. 32, no. 8, pp. 1200-1209, 1997.

[20] A. van Schaik and E. Fragniere, "Pseudo-voltage domain implementation of a 2-dimensional silicon cochlea," in Proc. Int. Symp. Circuits Syst. (ISCAS), Sydney, Australia, 2001, vol. 2, pp. 185-188.

[21] T. J. Hamilton, J. Craig, A. van Schaik, and J. Tapson, "A 2-D silicon cochlea with an improved automatic quality factor control-loop," in Proc. IEEE Int. Symp. Circuits Syst. (ISCAS), 2008, pp. 1772-1775.

[22] D. S. Freedman, H. I. Cohen, S. Deligeorges, C. Karl, and A. E. Hubbard, "An analog VLSI implementation of the inner hair cell and auditory nerve using a dual AGC model," IEEE Trans. Biomed. Circuits Syst., vol. 8, no. 2, pp. 240-256, Apr. 2014.

[23] A. Mohammad and P. D. Teal, "Using circuit analogies for analysis of cochlear models," Biomed. Eng. Lett., vol. 3, no. 4, pp. 263-272, 2013.

[24] M. Reit, W. Mathis, and R. Stoop, "Time-discrete nonlinear cochlea model implemented on DSP for auditory studies," in Proc. NDES Nonlinear Dyn. Electron. Syst., Wolfenbüttel, Germany, 2012.

[25] F. Gomez and R. Stoop, "Tuning the Hopf cochlea towards listening," in Proc. NDES Nonlinear Dyn. Electron. Syst., Wolfenbüttel, Germany, 2012.

[26] S. Wang, T. J. Koickal, A. Hamilton, E. Mastropaolo, R. Cheung, and L. Smith, "A floating active inductor based CMOS cochlea filter with high tunability and sharp cut-off," in Proc. IEEE Int. Symp. Circuits Syst. (ISCAS), 2013, pp. 193-196.

[27] R. Stoop, A. Kern, and J. J. van der Vyver, "How ears go electronic," in Proc. IEEE Conf. Nonlinear Theory Its Appl. (NOLTA), 2005.

[28] J. P. Van der Vyver, "A biomorphic electronic Hopf cochlea," Ph.D. dissertation, Diss. ETH, Zurich, Switzerland, 2006, no. 14915.

[29] A. Kern and R. Stoop, "Essential role of couplings between hearing nonlinearities," Phys. Rev. Lett., vol. 91, p. 128101, 2003.

[30] A. van Schaik and E. Fragniere, "Pseudo-voltage domain implementation of a 2-dimensional silicon cochlea," in Proc. Int. Symp. Circuits Syst. (ISCAS), Sydney, Australia, 2001, vol. 2, pp. 185-188.

[31] V. M. Eguluz, M. Ospeck, Y. Choe, A. J. Hudspeth, and M. O. Magnasco, "Essential nonlinearities in hearing," Phys. Rev. Lett., vol. 84, pp. $5232-5235,2000$ 
[32] S. Camalet, T. Duke, F. Jülicher, and J. Prost, "Auditory sensitivity provided by self-tuned critical oscillations of hair cells," Proc. Nat. Acad. Sci. USA, vol. 97, no. 7, pp. 3183-3188, 2000.

[33] J. Tapson, T. J. Hamilton, C. Jin, and A. van Schaik, "Self-tuned regenerative amplification and the Hopf bifurcation," in Proc. IEEE Int. Symp. Circuits Syst. (ISCAS), 2008, pp. 1768-1771.

[34] S. Gomar and A. Ahmadi, "Digital multiplierless implementation of biological adaptive-exponential neuron model," IEEE Trans. Circuits Syst. I, Reg. Papers, vol. 61, no. 4, pp. 1206-1219, Apr. 2013.

[35] E. M. Izhikevich, Dynamical Systems in Neuroscience: The Geometry of Excitability and Bursting. Cambridge, MA, USA: MIT Press, 2006.

[36] W. Gerstner and W. M. Kistler, Spiking Neuron Models Single Neurons, Populations, Plasticity. Cambridge, U.K.: Cambridge Univ. Press, Aug. 2002.

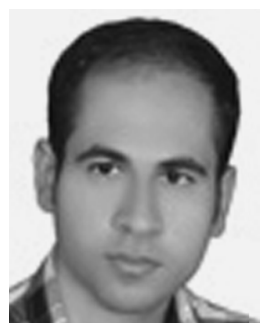

Moslem Nouri received the B.Sc. degree in electrical engineering from Azad University, Kermanshah, Iran, in 2012, and the M.Sc. degree in electronic engineering from the Department of Electrical Engineering, Razi University, Kermanshah, Iran, in 2014 (with Honors), where he is currently a Ph.D. student of electronic engineering, Razi University.

His research interests include high-frequency high-efficiency power amplifiers and oscillators, resonant $\mathrm{dc} / \mathrm{dc}$ power converters, numerical simulation of switching circuits, analog and digital electronic circuit design and optimization, bio-inspired computing, neuromorphic, artificial cochlear, and integrated circuit design.

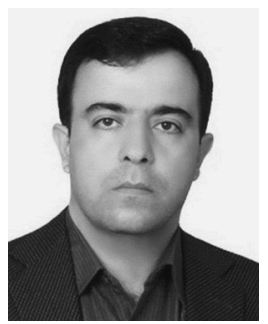

Arash Ahmadi (M'04) received the B.Sc. and M.Sc. degrees in electronics engineering from Sharif University of Technology and Tarbiat Modares University, Tehran, Iran, in 1993 and 1997, respectively, and the Ph.D. degree in electronics from the University of Southampton, U.K., in 2008.

He was with Razi University, Kermanshah, Iran, as a Faculty Member. From 2008 to 2010, he was a Fellow Researcher with the University of Southampton. He is currently an Associate Professor in the Electrical Engineering Department, Razi University. His current research interest include hardware implementation of signal processing systems, high-level synthesis, bio-inspired computing, and memristors.

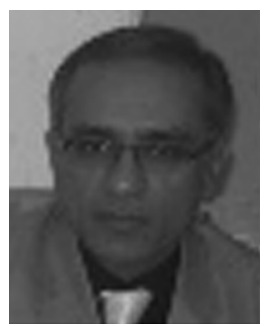

Shahpour Alirezaee received the B.Sc. in telecommunication engineering, M.Sc. and Ph.D. degrees in communication systems from Amirkabir University of Technology (Tehran Polytechnic), Tehran, Iran, respectively in 1996, 1998, and 2004. He has been with the Department of Electrical Engineering, University of Windsor, Windsor, ON, Canada, as a Visiting Scholar from 2004 to 2005. In 2005, he was with the Electrical Department, Faculty of Engineering, Razi University, Kermanshah, Iran. In January 2014, he joined University of Windsor as a Research Associate. His research interest includes wireless communication, wireless sensor networks, signal processing, and pattern recognition.

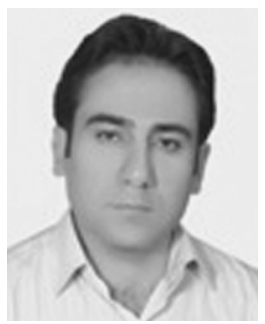

Gholamreza Karimi was born in Kermanshah, Iran, in 1977. He received the B.S. and M.S. and $\mathrm{PhD}$ degrees in electrical engineering from Iran University of Science and Technology (IUST) in 1999, 2001 and 2006 respectively. He is currently an Assistant Professor in Electrical Department at Razi University, Kermanshah, Iran, since 2007. His research interests include low power analog and digital IC design, RF IC design, modeling and simulation of RF mixed signal IC. He is also interested in microwave devices and artificial intelligence

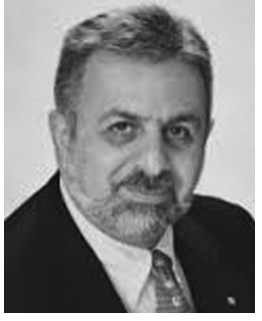

Majid Ahmadi (S'75-M'77-SM'84-F'02) received the B.Sc. degree in electrical engineering from Sharif University of Technology, Tehran, Iran, and the Ph.D. degree in electrical engineering from Imperial College, London University, London, U.K., in 1971 and 1977, respectively. Since 1980, he has been with the Electrical and Computer Engineering Department, University of Windsor, Windsor, ON, Canada, currently as a Professor and Director of the Research Center for Integrated Microsystems. He has coauthored the book Digital Filtering in 1-D and 2-Dimensions: Design and Applications (New York: Plennum, 1989) and has published more than 500 articles in the areas of digital signal processing, pattern recognition and computer vision, neural network architectures and applications, VLSI circuits and testing, and computer arithmetic. He is the Associate Editor of the Journal of Pattern Recognition and Regional Editor for the Journal of Circuits, Systems and Computers. His research interests include digital signal processing, MEMS, machine vision, pattern recognition, neural network architectures, and very large scale integration implementation.

Dr. Ahmadi is a Fellow of the IEE (U.K.). He was the IEEE Circuits and Systems Society Representative on the Neural Network Council and the Chair of the IEEE Circuits and Systems Neural Systems Applications Technical Committee. He received an Honorable Mention award from the Editorial Board of the Journal of Pattern Recognition in 1992 and the Distinctive Contributed Paper award from the Multiple-Valued Logic Conference Technical Committee and the IEEE Computer Society in 2000. He is a Fellow of IET (UK).

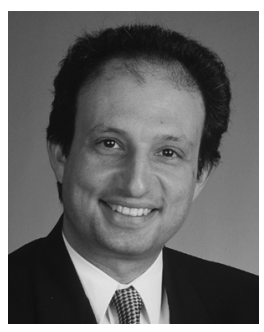

Derek Abbott (M'85-SM'99-F'05) was born in South Kensington, London, U.K., in 1960. He received the B.Sc. (honors) degree in physics from Loughborough University, Leicestershire, U.K., in 1982 and the Ph.D. degree in electrical and electronic engineering from the University of Adelaide, Adelaide, S.A. Australia, in 1995, under K. Eshraghian and B. R. Davis.

From 1978 to 1986, he was a Research Engineer at the GEC Hirst Research Centre, London, U.K. From 1986 to 1987, he was a VLSI Design Engineer at Austek Microsystems, Australia. Since 1987, he has been with the University of Adelaide, where he is presently a full Professor with the School of Electrical and Electronic Engineering. He coedited Quantum Aspects of Life (London, U.K.: Imperial College Press, 2008), coauthored Stochastic Resonance (Cambridge, U.K.: Cambridge University Press, 2012), and coauthored Terahertz Imaging for Biomedical Applications (New York, NY, USA: Springer-Verlag, 2012). He holds over 800 publications/patents and has been an invited speaker at over 100 institutions. His interests are in the area of multidisciplinary physics and electronic engineering applied to complex systems. His research programs span a number of areas of stochastics, game theory, photonics, biomedical engineering, and computational neuroscience.

Prof. Abbott is a Fellow of the Institute of Physics (IOP). He has won a number of awards including the South Australian Tall Poppy Award for Science (2004), the Premier's SA Great Award in Science and Technology for outstanding contributions to South Australia (2004), and an Australian Research Council (ARC) Future Fellowship (2012). He has served as an Editor and/or Guest Editor for a number of journals including the IEEE JOURNAL OF SOLIDState Circuits, Journal of Optics B, the Microelectronics Journal, Chaos, Smart Structures and Materials, Fluctuation and Noise Letters, PLOS ONE, and is currently on the editorial boards of the PROCEEDINGS OF THE IEEE, the IEEE PHOTONICS JOURNAL, and Nature's Scientific Reports. 